\title{
Penile transplantation: the US experience and institutional program set-up
}

\author{
April Adams Szafran, Richard Redett, Arthur L. Burnett \\ Johns Hopkins Medical Institutions, Baltimore, MD, USA \\ Contributions: (I) Conception and design: All authors; (II) Administrative support: All authors; (III) Provision of study materials or patients: All \\ authors; (IV) Collection and assembly of data: All authors; (V) Data analysis and interpretation: All authors; (VI) Manuscript writing: All authors; (VII) \\ Final approval of manuscript: All authors. \\ Correspondence to: Arthur L. Burnett, MD, MBA. Johns Hopkins School of Medicine, Brady Urological Institute, 600 North Wolfe Street, Marburg \\ 405, Baltimore, MD 21287, USA. Email: aburnet1@jhmi.edu.
}

\begin{abstract}
Penile transplantation using vascularized composite allografts is an emerging technique to treat genital loss. In the United States, this procedure has been performed successfully at Massachusetts General Hospital in a patient who had previously undergone treatment for penile cancer. The Johns Hopkins Medical Institutions has developed a research protocol to perform penile transplantation in patients with genital loss secondary to trauma. The process of selecting the appropriate candidate for genitourinary (GU) vascularized composite allograft surgery is rigorous including extensive physical examination, laboratory testing, imaging and psychological evaluations. After transplantation, limiting the potential complications associated with immunosuppression is critical given that the procedure is intended to improve quality of life and is not life-saving. Ultimately, penile transplant is a surgical intervention which may have numerous applications. Optimization of the pre-operative screening process, surgical technique, and immunosuppressive protocol is required to establish this method as the standard treatment for patients with genital loss and limited reconstructive options.
\end{abstract}

Keywords: Allotransplantation; genitourinary (GU); neophalloplasty

Submitted Dec 23, 2017. Accepted for publication Mar 05, 2018.

doi: 10.21037/tau.2018.03.14

View this article at: http://dx.doi.org/10.21037/tau.2018.03.14

\section{Introduction}

The initial concept for the development of a penile transplant program at the Johns Hopkins Medical Institutions began in earnest in November 2013. It was envisioned to be a multi-disciplinary approach led by The Department of Plastic and Reconstructive Surgery. Currently, the team includes physicians in infectious disease, cardiology and psychology as well as the surgical subspecialties including plastic surgery and urology. The initial aim was to restore functional status to combat war veterans who sustained devastating genital injuries with limited reconstructive options secondary to concomitant loss of donor sites that would normally be used for flap reconstruction (i.e., arms and legs). The reconstructive effort would be focused on using vascularized composite allografts, which would surpass many of the pitfalls of current penile reconstruction, particularly neophalloplasty. Specifically, conventional genital reconstruction has been plagued by poor cosmetic result, urethral fistula and/or stricture development, and inability to restore erectile function sufficiently as well as a requirement for multiple complex procedures.

The limitations of conventional neophalloplasty have been well established and the medical community is recognizing the need for an alternative method of reconstruction of the male genitalia. It has been estimated that 1,367 military servicemen had genital injuries between 2001-2013 while serving in Iraq and Afghanistan (1). Contemporary body armor results in increased survival, with many patients sustaining complex injuries requiring advanced reconstructive 
and prosthetic interventions (2). Current methods of reconstruction have failed to adequately restore the normal form and function of the penis. Furthermore, in the injured military population, both the radial forearm free flap and the pedicled anterolateral thigh flap for neophalloplasty may not be available to serve for reconstruction in patients who may have concomitant limb damage (1,3-5). Therefore, a significant need exists to provide effective therapy for these patients, especially when tissue loss is too extensive for local reconstructive efforts.

The indications for penile transplant may eventually reach beyond those initially outlined here. In addition to traumatic loss, individuals who may benefit from a genitourinary $(\mathrm{GU})$ transplant include: (I) patients undergoing treatment for penile cancer; (II) patients with congenital conditions resulting in a small or abnormal phallus; and (III) patients with gender dysmorphic syndrome transitioning from female to male. In these patients, the urologic concerns have been at the forefront of failed or problematic reconstructive efforts using conventional techniques such as neophalloplasty.

The theoretical principle for human penile allotransplantation has been established in a two-fold manner. Replantation of the penis after trauma using microsurgical techniques has demonstrated that when ischemic time is minimized, a functional and sensate phallus can be re-established. Vascularized composite allografts used for treatment of facial and limb deformity illustrate that these highly innervated tissues can be transferred to a recipient with both form and function being maintained when proper immunosuppression is administered (6-8). A combination of both techniques provides the framework for performing penile transplant surgery successfully.

The purpose of this review is to discuss the status of penile transplantation in the United States and to specifically outline the program at the Johns Hopkins Medical Institutions. The overall aim of the surgical procedure is to create a functional and aesthetic penis that allows for urination and restored sexual function while limiting the risks and complications of life-long immunosuppression.

\section{Review of penile transplantation in the US}

The first successful human penile transplant in the United States was performed at Massachusetts General Hospital in May 2016 on a patient who had undergone partial penectomy for treatment of penile cancer. Prior to this, here had been two other procedures of its kind performed in the world. The initial human penile transplant was undertaken in China on a 44-year-old male who had traumatic penile loss. The procedure was performed using a microsurgical technique, and the transplant itself was considered successful with immediate re-establishment of perfusion to the graft. There was no evidence of rejection during the short post-operative recovery period. The patient was maintained on a multi-drug immunosuppressive regimen consisting of mycophenolate, cyclosporine and prednisone. Postoperatively, the transplanted penis experienced venous congestion and discrete areas of skin necrosis. The graft was ultimately removed on post-operative day 14 due to psychological concerns raised by the patient and his wife $(9,10)$, although photos of the transplanted penis demonstrate what appears to be severe venous congestion. This failure highlighted that a rigorous pre-operative psychological evaluation is necessary before undergoing such an expansive surgical intervention. In addition to the operative and immunosuppressive considerations, patients would have to be vetted with regard to their psychological well-being and their acceptance of graft tissue from such an intimate area.

The second penile vascularized composite allograft procedure occurred in December 2014 in South Africa in a patient who had penile loss secondary to ritual circumcision complicated by gangrene. A comprehensive report of the procedure was described in the Lancet (11). The group's preparation, execution and the post-operative course with extended follow-up were described in detail. As part of the preparation for the transplant, the surgical team performed mock transplants in cadavers, which the group recommends to any hospital system attempting to start a penile transplant program (12). The recipient for the transplant was chosen from a pool of men 18 years and older who had total or partial penile loss secondary to ritual circumcision complications. The patient's outcomes were tracked using the 36 item Short Form survey, version 2 (SF-36v2) (13) and International Index of Erectile Function (IIEF) (14), standardized questionnaires which were completed before and after the intervention.

The South African patient and donor were matched for blood group and the recipient had no donor specific antibodies. After procuring the graft, the cold ischemia time was 16 hours. Due to the recipient's lack of a clear dorsal vein, the donor deep dorsal vein from the graft was anastomosed to the one of the deep inferior epigastric veins. The paired dorsal arteries of the penis were anastomosed 
to the left inferior epigastric artery and the left superficial external pudendal artery of the recipient. An end-toend, spatulated anastomosis was performed to join the donor and recipient urethras. Microsurgical technique was used to repair the nerves. The immunosuppressive protocol included a pre-operative dose of anti-thymocyte globulin and high doses steroids, followed by tacrolimus, mycophenolate and prednisone. The patient had penile rehabilitation with tadalafil daily for 3 months. Postoperative complications included the development of an infected hematoma and an urethrocutaneous fistula, both of which required further surgical intervention. At 24 months post procedure, the patient had improvement in his IIEF satisfaction score, mental health scores in the SF-36v2 and had a strong urinary stream. He reported erections sufficient for penetrative intercourse 5 weeks following the procedure. At 24 months post operatively, the patient had scores of $26 / 30$ in domain A, $8 / 10$ in domain $\mathrm{B}, 9 / 10$ in domain $\mathrm{C}$ and $12 / 15$ in domain $\mathrm{D}$, corresponding to erectile function, orgasmic function, sexual desire and intercourse satisfaction in the IIEF, respectively. The South African team has since performed another successful GU vascularized composite allograft, on a second patient disfigured by ritual circumcision.

The much anticipated details of the first male genital transplant performed in the United States with surgical specifics and the patient's post-operative course were published earlier this year (15). A high resolution computed tomography angiogram, diagnostic angiography and magnetic resonance imaging were performed preoperatively. These imaging studies clearly defined the vessels present within the penis: the dorsal artery of the penis, bulbar artery to the urethra, and the paired cavernosal arteries, which are responsible for filling the corpora cavernosa during erection. Two surgical teams were required, one to procure the donor allograft and a second to perform the reconstructive transplant in the recipient. Microsurgical technique was used with the addition of a harvested vein graft to facilitate the arterial and venous anastomoses. Additionally, a cadaveric acellular nerve allograft was used as part of the neurorrhaphies. The immunosuppressive regimen included anti-thymocyte globulin, mycophenolate, and methylprednisolone preoperatively, followed by tacrolimus, mycophenolate, and a steroid taper post-operatively.

The patient required two re-operations, one for hematoma evacuation on post-operative day 2 and a second for skin debridement on post-operative day 13. The patient had an acute rejection episode on post-operative day 28 that required high dose methylprednisolone followed by antithymocyte globulin combined with high dose steroids and an oral steroid taper. At 6 months following the procedure, the patient reports sensation at the proximal penile shaft and good urinary stream. The erectile function is described qualitatively as partial spontaneous erections which are increasing in both quality and frequency. Overall, penile transplantation appears to be a successful procedure with high patient satisfaction. Some of the main goals of $\mathrm{GU}$ vascularized composite allotransplantation have been achieved in the case reports currently described in the literature. In both the South African and US penile transplants restoration of urination and erectile functions as well as return of sensation have been demonstrated to various degrees in these patients.

\section{Program components, Johns Hopkins Hospital}

\section{Pre-operative screening}

The transplant program at Johns Hopkins has focused on treating wounded warriors who have at least $75 \%$ genital loss from combat injuries with limited reconstructive options due to concomitant extremity injuries. Funding for the transplant is provided primarily by institutional funds available to support the surgical procedure. Potential candidates have been identified by reviewing our patient population at the Johns Hopkins Hospital and through referrals from the United States military. The screening process includes an initial telephone interview followed by a comprehensive clinical history and physical examination performed by the principal investigator. This is further augmented by a battery of laboratory tests, a comprehensive psychological evaluation and imaging studies.

We standardly obtain the following laboratory tests: complete blood count with differential, complete metabolic panel, evaluation of clotting factors, liver function tests, hemoglobin A1c, alpha fetoprotein, carcinoembryonic antigen, electrolytes, blood typing and screen, tissue typing, urine toxicology screen, urinalysis, infectious disease studies (viral testing including Human Immunodeficiency Virus, Epstein-Barr Virus , Cytomegalovirus and Herpes Simplex Virus screening as well as testing for syphilis, tuberculosis and toxoplasmosis). Imaging studies include but are not limited to: chest X-ray, GU MR neurography, CT angiography, pelvic $\mathrm{X}$-rays and sinus films, the latter to rule out subclinical infection. In addition to the 
Table 1 Pre-operative screening evaluation

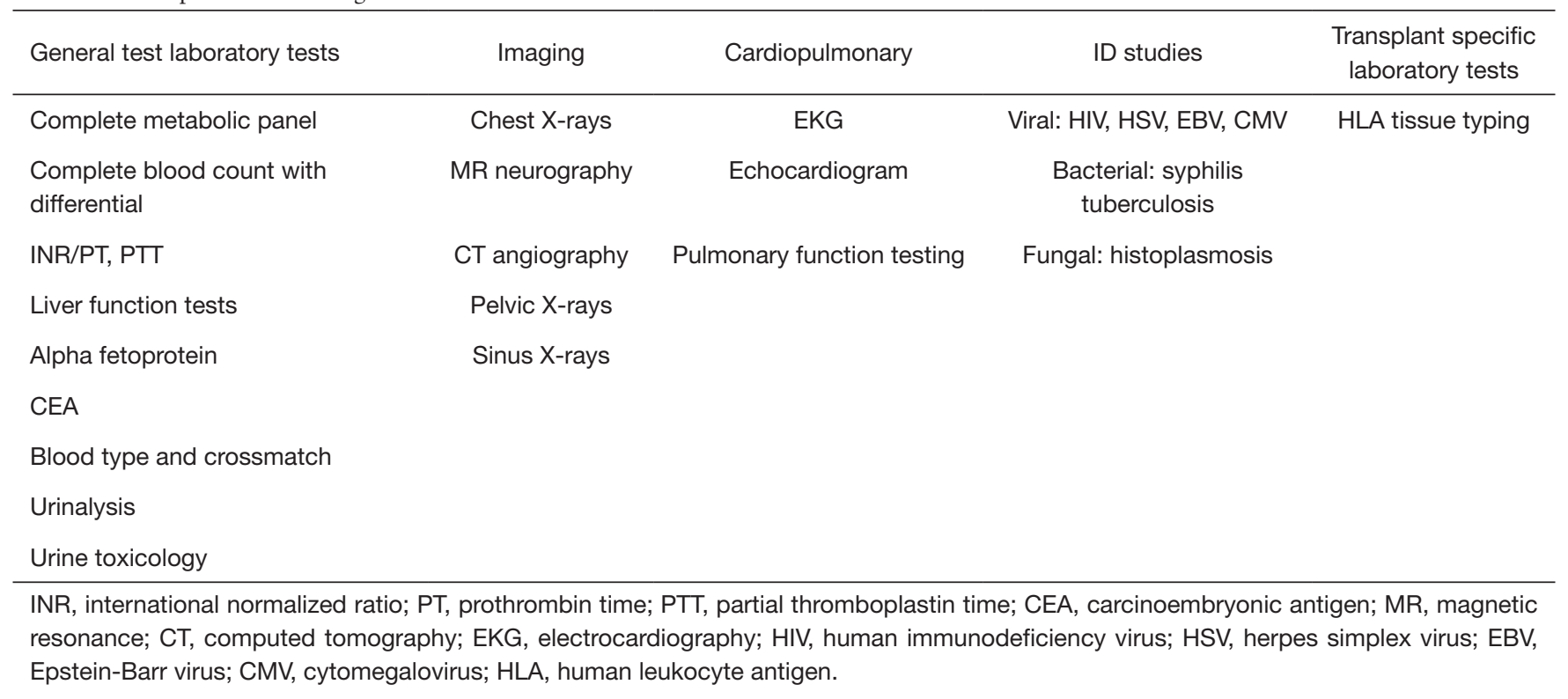

above, other adjuvant studies that are standardly obtained include electrocardiography (EKG), echocardiogram, and pulmonary function tests. Colonoscopy and esophagogastroduodenoscopy are additional procedures to be performed if clinically indicated (Table 1).

In addition to the initial evaluation by the principal investigator in the Department of Plastic and Reconstructive Surgery, the patient must be examined in consultation by the following specialties: urology (with examination of the prostate as indicated and completion of the IIEF and Erection Hardness Score) (16), cardiology, infectious disease, psychiatry and ophthalmology (general vision screen and evaluation for diabetic retinopathy). The patient requires a dental exam as standard screening. The psychiatric evaluation is extensive and includes an assessment of the patient's mental state, general cognitive function, coping mechanisms, and an evaluation of the psychological impact of penile trauma as well as the patient's mental preparedness to receive a cadaveric penile graft. If the patient is in a relationship, the partner is also included in the evaluation.

The patient completes the Mini-Mental Status Exam (17), the Hopkins Adult Reading Test (18), Hopkins Verbal Learning Test (19), Recognition Memory Test (20) and a Brief Visuospatial Memory Test (21). The psychological measures include the Brief Symptom Inventory (22), the Affect Balance Scale (23), and NEO Five-Factory Inventory (24) which is completed by a significant other or a close family member. Psychosexual quality of life Questionnaires will be administered which include Self-Esteem and Relationship (25) as well as the Modified Sexual Life Quality Questionnaires (26). A transplant social worker participates in the psychological assessment to confirm that the patient will have the appropriate support system following transplant.

\section{Proposed surgical procedure}

At the Johns Hopkins Medical Institutions, the transplanted graft would be limited to non-germline tissues and would potentially include the penis, scrotum, adjacent skin, muscle and potentially a portion of the pubic bone. When a suitable donor is available and the family has been consented, the graft will be procured by the donor retrieval team. The recipient would be notified and transported to the transplant center.

The graft would be wrapped in a moist sterile gauze at a temperature of 4-6 degrees centigrade for transport. The donor spleen, lymph nodes and spinal column bone marrow would also be procured and cryopreserved to be used as part of the immunosuppressive protocol that requires infusion of donor bone marrow on post-operative day 14. Much of the specifics of surgical technique would be dependent on both the donor and recipient anatomies. The surgical team has performed cadaveric GU transplant procedures as a method of training for the actual operative day. Lessons learned during these cadaveric trials illustrate that performing the 

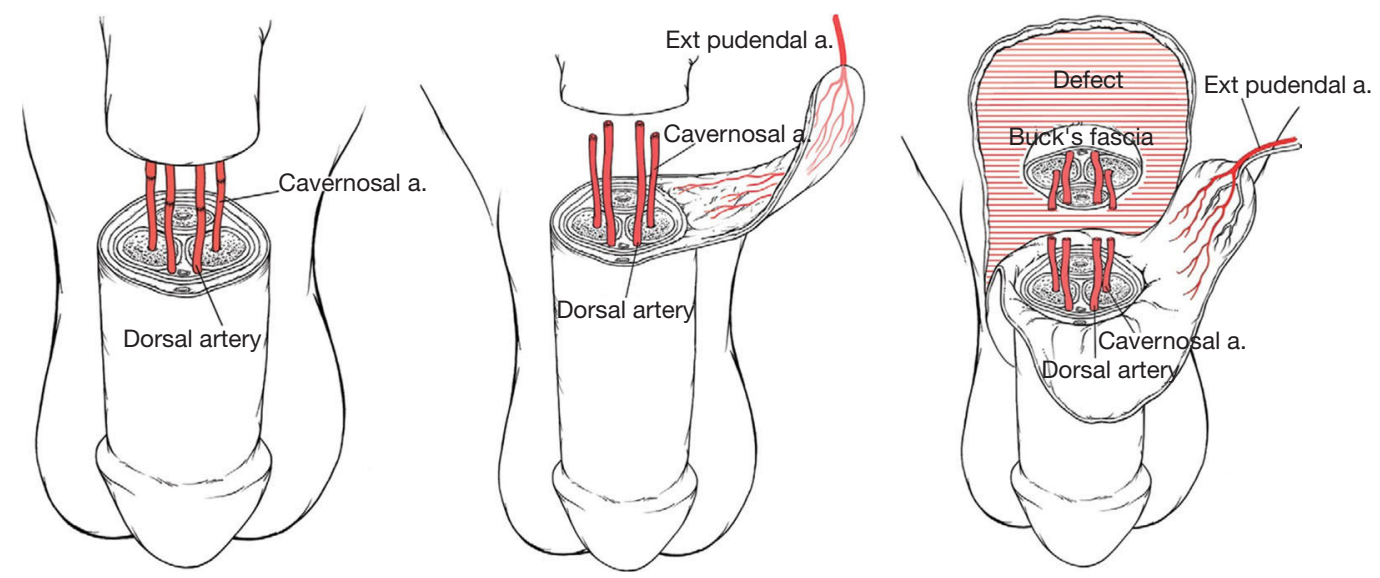

Figure 1 Depiction of vascular anastomoses to provide optimal blood flow to penile graft (27).

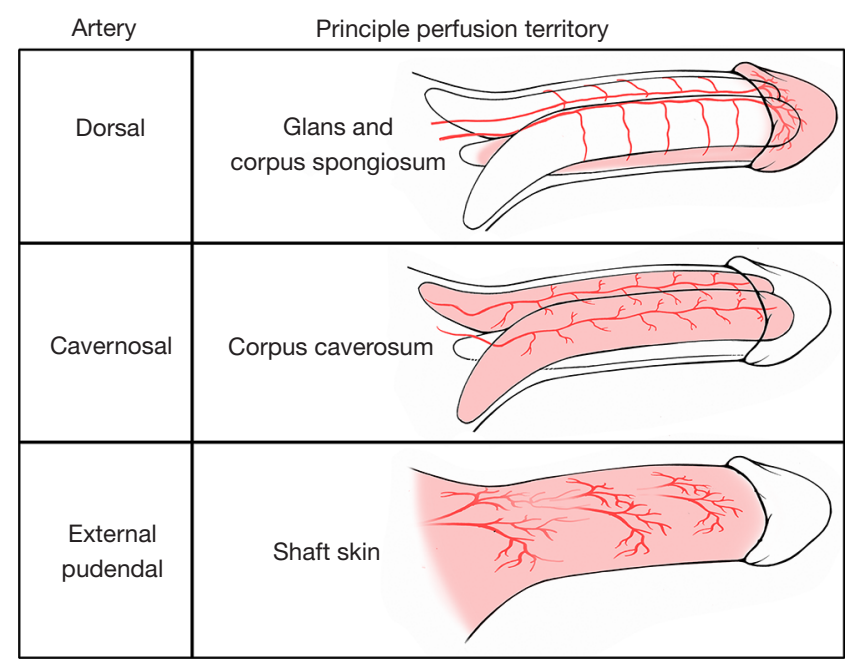

Figure 2 Primary perfusion territories of the dorsal, cavernosal, and external pudendal arteries (27).

cavernosal anastomoses may improve later erectile function in the recipient without the requirement for additional surgery to place a penile prosthetic device. Also, use of the external pudendal artery may avoid the complication of penile shaft skin necrosis as this vessel was demonstrated to provide much of the vascularization to this region of the graft (Figures 1,2) (27).

\section{Immunosuppressive regimen}

There is no doubt that the possible side effects of high dose immunosuppression, including infection and renal toxicity, are accepted consequences of life-saving solid organ transplants. For vascularized composite allografts, the surgery's main aim is to improve or enhance quality of life and to restore a sense of self for the patients. A primary effort of the research protocol at the Johns Hopkins Medical Institutions for penile transplantation is to limit immunosuppression. The immunosuppressive protocol would consist of monoclonal antibody induction therapy with a humanized anti CD52 agent, donor bone marrow infusion at day 14 post-transplant, followed by tacrolimus only immunosuppression. The purpose of the bone marrow infusion is to increase donor tolerance to the grafted tissue. Frequent biopsies of the graft are to be staged at specific intervals to pick up early rejection. After the graft has been stably accepted attempts will be made to wean tacrolimus/ FK506. The choice of this agent, which is a calcineurin inhibitor that suppresses interleukin-2 synthesis and $\mathrm{T}$ cell function, extends beyond its immunosuppressive abilities (28). In an ex vivo model to evaluate both immune rejection and the effects of immunosuppressive medications on cavernosal tissue, cyclosporine was shown to prevent cavernous tissue relaxation. This negative effect on cavernosal tissue physiology was not seen in tissues exposed to FK506 (29). Additionally, in animal studies, tacrolimus has been shown to enhance repair of damaged peripheral nerves (30). The combination of preserving erectile tissue function as well as enhancing neuronal repair make FK506 an ideal immunosuppressive agent in the setting of $\mathrm{GU}$ vascularized allotransplantation surgery.

\section{Post-operative assessments}

The transplant recipients are to be followed in the 
Transplant Clinic for their entire life following the transplant. The patients will be screened for possible rejection of the graft by physical examination, tissue biopsies as indicated, and evaluation for donor specific antibodies. The patients will have blood tests drawn regularly to monitor tacrolimus levels as well as renal function. Tests for urinary flow as well as standardized questionnaires including the IIEF and the Erection Hardness Scale will be obtained to monitor progress from a GU perspective.

After reviewing the literature and our own experience developing a $G U$ vascularized composite allograft program at Johns Hopkins Medical Institutions, we have the following recommendations for consideration. A rigorous pre-operative screening should be performed which includes laboratory testing as well as an extensive psychological evaluation. Due to variations in patient anatomy, comprehensive imaging should be obtained preoperatively to evaluate patient vasculature and neural innervation. With regard to the procedure itself, surgeons should invest time in simulating the surgery using cadavers prior to the actual initial surgery and care should be taken to establish multiple channels of revascularization via cavernosal, dorsal and external pudendal arteries to provide the most functional phallus and to potentially avoid complications like skin necrosis. The optimal immunosuppressive regimen has not yet been established. We, however, recommend tacrolimus as part of the protocol given its potential to improve nerve regeneration and to maintain normal physiology of penile erectile tissue. Finally, as penile transplant becomes more frequently performed, a consensus statement should be developed to guide the field.

\section{Conclusions}

Surgical treatment of traumatic or iatrogenic penile loss remains difficult. The issues lie mainly in the limitations of current reconstructive efforts, which rely on tissues that fail to recapitulate the many functions of an adult male phallus. The result is patient dissatisfaction and multiple postoperative complications requiring complex repairs. Penile transplant, while in its infancy, promises improved overall surgical outcomes with regard to cosmesis and function, as well as limiting the need for repeat highly complex surgical re-interventions. Educating the public and overcoming bias against donation of $\mathrm{GU}$ tissue for vascularized composite allograft transplantation will be a major hurdle to the success of this treatment modality $(31,32)$. At the Johns Hopkins Medical Institutions, there is an ongoing effort to identify ideal candidates to participate in the GU vascularized composite allotransplantation protocol. Due diligence is required now and in the future to transform this technique into the standard of care for the treatment of men with traumatic loss.

\section{Acknowledgements}

Jane Littleton, CRNP, is the Vascularized Composite Allotransplantation Coordinator. She provided information to review regarding the Penile Transplant Program at the Johns Hopkins Medical Institutions and discussed the initial screening process. This work is supported by the Johns Hopkins Medical Institutions.

\section{Footnote}

Conflicts of Interest: The authors have no conflicts of interest to declare.

\section{References}

1. Janak JC, Orman JA, Soderdahl DW, et al. Epidemiology of Genitourinary Injuries among Male U.S. Service Members Deployed to Iraq and Afghanistan: Early Findings from the Trauma Outcomes and Urogenital Health (TOUGH) Project. J Urol 2017;197:414-9.

2. Prat N, Rongieras F, Sarron JC, et al. Contemporary body armor: technical data, injuries, and limits. Eur J Trauma Emerg Surg 2012;38:95-105.

3. Sopko NA, Tuffaha SH, Lough D, et al. Penile Allotransplantation for Complex Genitourinary Reconstruction. J Urol 2017;198:274-80.

4. Sopko NA, Burnett AL. Penile transplantation is here. Lancet 2017;390:1008-10.

5. Tuffaha SH, Cooney DS, Sopko NA, et al. Penile transplantation: an emerging option for genitourinary reconstruction. Transpl Int 2017;30:441-50.

6. Segal RL, Massanyi EZ, Gupta AD, et al. Inflatable penile prosthesis technique and outcomes after radial forearm free flap neophalloplasty. Int J Impot Res 2015;27:49-53.

7. Massanyi EZ, Gupta A, Goel S, et al. Radial forearm free flap phalloplasty for penile inadequacy in patients with exstrophy. J Urol 2013;190:1577-82.

8. Leriche A, Timsit MO, Morel-Journel N, et al. Long-term outcome of forearm flee-flap phalloplasty in the treatment of transsexualism. BJU Int 2008;101:1297-300.

9. $\mathrm{Hu} \mathrm{W,} \mathrm{Lu} \mathrm{J,} \mathrm{Zhang} \mathrm{L,} \mathrm{et} \mathrm{al.} \mathrm{A} \mathrm{preliminary} \mathrm{report} \mathrm{of} \mathrm{penile}$ 
transplantation. Eur Urol 2006;50:851-3.

10. $\mathrm{Hu} \mathrm{W}, \mathrm{Lu} \mathrm{J}$, Zhang $\mathrm{L}$, et al. A preliminary report of penile transplantation: part 2. Eur Urol 2006;50:1115-6; discussion 1116.

11. van der Merwe A, Graewe F, Zühlke A, et al. Penile allotransplantation for penis amputation following ritual circumcision: a case report with 24 months of follow-up. Lancet 2017;390:1038-47.

12. Merwe AV, Zarrabi A, Zühlke A, et al. Lessons learned from the world's first successful penis allotransplantation. J Mater Sci Mater Med 2017;28:27.

13. Brazier JE, Harper R, Jones NM, et al. Validating the SF36 health survey questionnaire: new outcome measure for primary care. BMJ 1992;305:160-4.

14. Rosen RC, Riley A, Wagner G, et al. The international index of erectile function (IIEF): a multidimensional scale for assessment of erectile dysfunction. Urology 1997;49:822-30.

15. Cetrulo CL Jr, Li K, Salinas HM, et al. Penis Transplantation: First US Experience. Ann Surg 2018;267:983-88.

16. Mulhall JP, Goldstein I, Bushmakin AG, et al. Validation of the erection hardness score. J Sex Med 2007;4:1626-34.

17. Folstein MF, Folstein SE, McHugh PR. "Mini-mental state". A practical method for grading the cognitive state of patients for the clinician. J Psychiatr Res 1975;12:189-98.

18. Schretlen DJ, Winicki JM, Meyer SM, et al. Development, psychometric properties, and validity of the Hopkins adult reading test (HART). Clin Neuropsychol 2009;23:926-43.

19. Hogervorst E, Combrinck M, Lapuerta P, et al. The Hopkins Verbal Learning Test and screening for dementia. Dement Geriatr Cogn Disord 2002;13:13-20.

20. Kim MS, Boone KB, Victor T, et al. The Warrington Recognition Memory Test for words as a measure of response bias: total score and response time cutoffs developed on "real world" credible and noncredible subjects. Arch Clin Neuropsychol 2010;25:60-70.

21. Tam JW, Schmitter-Edgecombe M. The role of processing

Cite this article as: Szafran AA, Redett R, Burnett AL. Penile transplantation: the US experience and institutional program set-up. Transl Androl Urol 2018;7(4):639-645. doi: 10.21037/ tau.2018.03.14 speed in the Brief Visuospatial Memory Test-Revised. Clin Neuropsychol 2013;27:962-72.

22. Boulet J, Boss MW. Reliability and validity of the Brief Symptom Inventory. Psych Assessment 1991;3:433-7.

23. Perkinson MA, Albert SM, Luborsky M, et al. Exploring the Validity of the Affect Balance Scale with a sample of family caregivers. J Gerontol 1994;49:S264-75.

24. Scandell DJ. Development and initial validation of the validity scales for the NEO-Five Factor Inventory. Pers Individ Dif 2000;29:1153-62.

25. Cappelleri JC, Althof SE, Siegel RL, et al. Development and validation of the Self-Esteem and Relationship (SEAR) questionnaire in erectile dysfunction. Int J Impot Res 2004;16:30-8.

26. Woodward JM, Hass SL, Woodward PJ. Reliability and validity of the sexual life quality questionnaire (SLQQ). Qual Life Res 2002;11:365-77.

27. Tuffaha SH, Sacks JM, Shores JT, et al. Using the dorsal, cavernosal and external pudendal arteries for penile transplantation: Technical considerations and perfusion territories. Plast Reconstr Surg 2014;134:111-9e.

28. Chinen J, Buckley RH. Transplantation immunology: solid organ and bone marrow. J Allergy Clin Immunol 2010;125:S324-35.

29. Sopko NA, Matsui H, Lough DM, et al. Ex Vivo Model of Human Penile Transplantation and Rejection: Implications for Erectile Tissue Physiology. Eur Urol 2017;71:584-93.

30. He XZ, Ma JJ, Wang HQ, et al. Brain injury in combination with tacrolimus promotes the regeneration of injured peripheral nerves. Neural Regen Res 2017;12:987-94.

31. Campbell JD, Burnett AL. Moving towards successful penile transplantation programmes. Nat Rev Urol 2018;15:75-6.

32. Rodrigue JR, Tomich D, Fleishman A, et al. Vascularized composite allograft donation and transplantation: A survey of public attitudes in the United States. Am J Transplant 2017;17:2687-95. 